\title{
The birth-cluster of the galactic luminous blue variable WRA $751^{\star}$
}

\author{
A. Pasquali ${ }^{1,2}$, F. Comerón ${ }^{3}$, and A. Nota ${ }^{4}$ \\ 1 Institute of Astronomy, ETH Hönggerberg, HPF, 8093 Zürich, Switzerland \\ 2 Max-Planck-Institut für Astronomie, Königstuhl 17, 69117 Heidelberg, Germany \\ e-mail: pasquali@mpia.de \\ 3 ESO, Karl-Schwarzschild-Strasse 2, 85748 Garching bei München, Germany \\ e-mail: fcomeron@eso.org \\ 4 STScI, 3700 San Martin Drive, MD28201 Baltimore, USA \\ e-mail: nota@stsci.edu
}

Received 3 August 2005 / Accepted 8 November 2005

\begin{abstract}
We present the results of NTT/VLT $U B V$ imaging of a $260 \mathrm{arcmin}^{2}$ region containing the Galactic Luminous Blue Variable WRA 751, in search for its birth-cluster, i.e. a cluster of young and massive stars spatially and physically associated with it. On the basis of the classical reddeningfree parameter $Q$, we have identified a sample of 24 early-type stars with colours typical of spectral types earlier than B3. Interestingly, these stars are clustered within a radius of $1^{\prime}$ from WRA 751, corresponding to about $1 \%$ of the imaged field. These stars tightly distribute around $(B-V) \simeq 1.67$, which in turn defines a mean extinction $A_{\mathrm{V}} \simeq 6.1$ mag. The 5 brighter $(V \leq 16.2)$ and bluer $(Q \leq-0.9)$ stars of the sample have been subsequently observed with FORS1 and classified as 3 late O- and 2 early B-stars. The absence of stars earlier than O8 indicates an age of the cluster older than $4 \mathrm{Myr}$, although it could be due to an incomplete sampling of the upper end of the main sequence. Nevertheless, the detection of $\mathrm{OB}$ stars of class I certainly indicates an age of a few million years. At an assumed distance of $6 \mathrm{kpc}$, we estimate a cluster radius of $3.4 \mathrm{pc}$ and a total mass of $2.2 \times 10^{3} M_{\odot}$. Our discovery is only the second known instance of a Galactic Luminous Blue Variable associated with its birth-cluster.
\end{abstract}

Key words. stars: early-type - stars: evolution - stars: fundamental parameters - stars: Hertzsprung-Russell (HR) and C-M diagrams

\section{Introduction}

Massive stars $\left(M_{i} \geq 40-60 M_{\odot}\right.$, i.e. progenitors of supernovae of type II) play a key role in galaxy formation and evolution. Their stellar winds and end-of-life explosions set the kinematics and chemistry of the interstellar medium, and power galactic fontains and outflows, which, in turn, significantly affect galaxy evolution (i.e. feedback, Dekel \& Silk 1986). This is true at any lookback time; however, beyond the Local Group, individual stars are no longer resolved and one only observes their effect integrated over the entire galaxy. To interpret these observations, population synthesis codes are required (i.e. Bruzual \& Charlot 2003; Leitherer et al. 1999). The accuracy of these models relies on our detailed knowledge of the first step in the ladder, the massive stars evolution, and, in particular, the dependence of mass loss on initial stellar mass and time (cf. Langer et al. 1994). This is still under debate.

^ Based on observations obtained with ESO telescopes on La Silla and Paranal, Chile, under programmes 271.D-5008, 71.C-0432, and 073.D-0037.
Theoretical predictions indicate that mass loss is a competition among stellar gravity, radiation pressure and rotation (Maeder \& Meynet 2000). Unfortunately, observations have failed so far to quantitatively constrain the rotational velocity of evolved massive stars. Mass loss becomes extreme in the post-main sequence evolution, when stars reduce their mass by a factor of $\geq 4$ and approach the final stage of supernova. It is believed that massive stars remove the majority of their envelope during the Luminous Blue Variable (LBV) phase, via giant eruptions that produce circumstellar, chemically-enriched nebulae (i.e. Eta Carinae, Humphreys \& Davidson 1994).

Nebular expansion velocities and nebular sizes set the lifetime of the LBV phase to $\simeq 10^{4} \mathrm{yr}$ (Nota 1997), and this is the reason why only about 30 candidate and confirmed LBV stars are known in the entire Local Group (Humphreys \& Davidson 1994; Nota 1997). Of these, eleven belong to the Milky Way and only one confirmed LBV is located in a star cluster (Eta Carinae in Trumpler 14/16, Massey et al. 2001), and a candidate LBV candidate, VI Cygn No. 12 is associated with Cgynus OB2. In the LMC, only one confirmed LBV out of 10 
is a member of a cluster of massive stars (S Doradus in LH 41, Massey et al. 2000).

The presence of a cluster of $\mathrm{OB}$ stars associated with an LBV star gives us the possibility to infer the distance, age and mass of the LBV progenitor by fitting the cluster ZeroAge Main sequence and its turn-off. In this way, Massey et al. (2001) have derived a progenitor mass of about $120 M_{\odot}$ and an age of about 1 Myr for both LBV stars. The authors have thus suggested that LBVs are a normal stage in the evolution of the most massive stars.

To further test this hypothesis better statistics are required, and the discovery of the birth-cluster of a LBV star is thus extremely valuable. In this paper we report on the discovery of a second case of association between a confirmed galactic LBV and an OB cluster. The LBV star is WRA 751 in the Galactic Carina arm. Long-slit spectra of its circumstellar nebula (Nota, private communication) revealed the presence of three emission-line (i.e. $\mathrm{H} \alpha$ ) stars in the close vicinity of WRA 751. Unfortunately, the spectra only covered the wavelength range 6200-8000 , and the 4000-5000 $\AA$ interval, needed to derive reliable spectral types, was missing. We have therefore followed-up these early observations with $U B V$ imaging of a region centred on WRA 751 to identify other possible young, massive stars on the basis of their $U B V$ photometry. We do confirm the existence of moderately reddened earlytype stars and present spectra of a selection of them, which we classify as late O- and early B-type stars of various luminosity classes. The absence of stars earlier than $\mathrm{O} 8$ indicates a cluster age older than 4 Myr.

The observations and data reduction are presented in Sect. 2, while the identification of the WRA 751 cluster is described in Sect. 3. The photometric and spectroscopic properties of the cluster stars are discussed in Sect. 4, and conclusions follow in Sect. 5.

\section{Observations}

\subsection{Imaging}

Our first set of imaging observations was obtained on the night of 1/2 April 2003 using EMMI, the visible imager and spectrograph at the ESO New Technology Telescope on La Silla. The observations were designed so as to include the region immediate to WRA 751 as well as an extended, diffuse nebulosity visible in the UK Schmidt Telescope H $\alpha$ survey (Parker 1997) extending to its Southwest. An area of $18.6 \times 11{ }^{\prime} .4$ centered on the coordinates $\alpha(2000)=11^{\mathrm{h}} 08^{\mathrm{m}} 15^{\mathrm{s}} 5, \delta(2000)=-60^{\circ} 47^{\prime} 35^{\prime \prime}$ was covered in a mosaic of pointings through the $U, B$, and $V$ filters. The $U$ and $B$ frames were obtained on the EMMI blue arm, which uses a $1024 \times 1024$ pix $^{2}$ detector yielding a field of view of $6.2 \times 6.2$ per frame with a scale of $0^{\prime} .37 \mathrm{pix}^{-1}$. The $V$ frames were obtained with the EMMI red arm, which uses an array of two $2048 \times 4096$ pix $^{2}$ detectors yielding a field of view of $9^{\prime} .1 \times 9$.' 9 . We used $2 \times 2$ binning in the red arm, resulting in a scale of $0.333 \mathrm{pix}^{-1}$. The field was covered in a sequence of ten pointings in each filter consisting of telescope offsets of $3^{\prime}$ westwards, followed by offsets of $5^{\prime}$ alternatively northwards and southwards. The offsets ensured a broad overlap between adjacent frames resulting in each sky position being recorded by at least two ( $U$ and $B$ filters) or three ( $V$ filter) pixels. Exposure times for each frame were $120 \mathrm{~s}(U), 45 \mathrm{~s}(B)$, and $10 \mathrm{~s}(V)$. Additional observations were obtained of the Landolt (1992) standard field around PG1047+003 in order to determine the photometric zeropoints in each filter. Characteristic extinction coefficients for La Silla were taken from the observatory Web pages, and photometric system transformation coefficients were obtained from the EMMI User Manual.

The NTT observations were reduced using standard IRAF ${ }^{1}$ tasks to carry out the usual steps of bias subtraction, flat-field correction using twilight sky flat field frames, removal of cosmic ray hits, and construction of a bad pixel mask based on the pixel value histograms of bias and flat field frames. Precise offsets among the images composing the mosaics were determined using the position of the numerous stars in the overlap areas between consecutive frames as a reference. The images were then combined into a single mosaic of the field by averaging the pixel values at each sky position, discarding those flagged in the bad pixel mask. Finally, the mosaics obtained in each filter were registered and cropped to the common area.

A second set of observations was obtained using FORS2, one of the visible imagers and low-resolution spectrograph instruments at the ESO Very Large Telescope, in Service Mode on the night of 7/8 March 2004. The instrument detector is composed by mosaic of two $4096 \times 2048$ pix $^{2}$ detectors yielding a pixel scale of 0.253 pix $^{-1}$ with the $2 \times 2$ binning used. A dither pattern focusing on the northern region of the WRA 751 cluster, which was not included in our previous NTT observations, was observed through the $U B V$ filters. We used the pipeline-reduced individual images included by the Observatory in the data package (Silva \& Péron 2004) and stacked them into a single frame per filter for a given exposure time. Two exposure times per filter were used: $5 \mathrm{~s}$ and $120 \mathrm{~s}(U)$, and $1 \mathrm{~s}$ and $60 \mathrm{~s}(B, V)$, so as to increase the dynamic range by obtaining "shallow" and "deep" images. The field covered by the FORS 2 observations is 7'.06 $\times 7$ '.06 centered on $\alpha(2000)=11^{\mathrm{h}} 08^{\mathrm{m}} 40^{\mathrm{s}} 4, \delta(2000)=-60^{\circ} 42^{\prime} 51^{\prime \prime}$. Photometric calibration was achieved by using calibration plan observations of a Landolt (1992) standard field in the SA 100 area containing star \#269, also reduced by the FORS2 pipeline and included in the data package. Extinction and colour coefficients were taken from the FORS2 Quality Control Web pages maintained by ESO (http: //www . eso.org/observing/dfo/quality/).

Instrumental photometry was carried out under IRAF by means of dedicated scripts that make use of tasks in the DAOPHOT package (Stetson 1987). Sources were first detected with DAOFIND, using first the images of isolated, unsaturated bright stars to determine an approximate point-spread function (PSF), needed for the identification of point sources. Photometry of all the stars detected by DAOFIND in each frame was then performed by defining an undersized aperture having as a radius the full-width at half maximum of the PSF of the reference star, and measuring the flux inside it at the

1 IRAF is distributed by NOAO, which is operated by the Association of Universities for Research in Astronomy, Inc., under contract to the National Science Foundation. 
position of each detected object using the PHOT task under DAOPHOT. The rest of the flux in the PSF of each star was then added by fitting a circularly symmetric radial profile to the distribution of counts of each star outside the aperture, rather than by using a constant aperture correction. This procedure allowed us to remove the photometric contamination due to other stars located on the wings of the PSF, and to adjust to the mild variations of the image quality across the combined images. For the FORS2 observations, separate catalogs of magnitudes for each filter were produced from the deep and shallow images, and the magnitudes were then compared to determine the onset of the non-linearity regime of the detector. The magnitude of each star was taken from the measurement in the deep image unless its magnitude was less than 0.5 mag fainter than the start of the non-linearity regime. For brightest stars the measurement on the shallow image was preferred instead.

A merged catalog containing the results from the EMMI and FORS 2 observations was finally prepared. The magnitudes measured with FORS2 were preferred when available due to their higher quality, and the EMMI magnitudes were used in the area not covered by the VLT observations.

\subsection{Spectroscopy}

The spectroscopic observations were performed in Service Mode with the FORS1 instrument on the VLT using multiobject spectroscopy (MOS) on the night of 3/4 July 2003. A selection of targets was made from our EMMI observations (which were the only ones available at the time) based on magnitudes brighter than $V \simeq 16.2$ and colours bluer than $Q \simeq-0.9$. The MOS masks in FORS1 are defined by a set of movable blades, which constrains the number of slits that can be placed on a small area of the sky such as that subtended by the WRA 751 cluster. With this setup we could obtain spectra of five OB star candidates (located within 1' from WRA 751 itself) in a single $600 \mathrm{~s}$ exposure. Spectra were taken through the GRIS_600B+12 grism covering the spectral range between $3450 \AA$ and $5900 \AA$, where the diagnostic H, He, C and $\mathrm{N}$ lines used in the MK spectral type classification scheme lie. The GRIS_600B+12 grism is characterised by a dispersion of $1.2 \AA /$ pix and a FWHM resolution of $4.8 \AA$.

The data were reduced with the standard routines in the IRAF APEXTRACT and ONEDSPEC packages.

\section{Identifying blue stars}

At a galactic longitude $l \simeq 290^{\circ}$.7 our field probes a line of sight providing a nearly end-on view of the Sagittarius-Carina arm, which extends between $\sim 1.5 \mathrm{kpc}$ and $\sim 8 \mathrm{kpc}$ in that direction (e.g. Georgelin \& Georgelin 1976). At the low galactic latitude of the field, $b \simeq-0{ }^{\circ} 4$, the far end of the arm lies less than $60 \mathrm{pc}$ from the galactic plane. It is thus expected to find in our field stars with highly varying degrees of extinction, leading us to adopt reddening-free selection criteria to identify the earliesttype stars in the region.

Figure 1 shows the $(B-V),(U-B)$ diagram of all the non-saturated stars in the field with colours determined to better than $\sigma(B-V), \sigma(U-B)=0.2$. The unreddened main

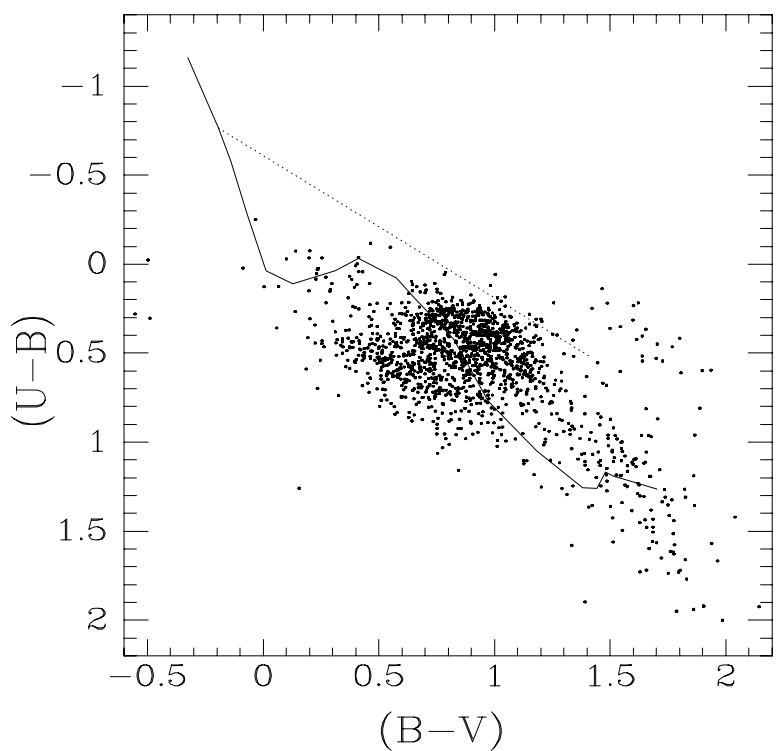

Fig. 1. $U B V$ colour-colour diagram showing the positions of the nonsaturated stars in our field having $\sigma(B-V), \sigma(U-B)<0.2$. The solid line is the locus of unreddened main sequence stars between spectral types O and M 5 (Bessell 1990). The dashed line is a reddening vector whose length corresponds to a visible extinction $A_{\mathrm{V}}=5 \mathrm{mag}$.

sequence locus from Bessell (1990) is plotted, as well as a reddening vector showing the displacement of a B3V star obscured by $A_{\mathrm{V}}=5$ mag with a reddening law characterized by $A_{\mathrm{V}}=3.1 E[B-V], E[U-B] / E[B-V]=0.80$. The adopted reddening ratio $E[U-B] / E[B-V]$, also preferred by Massey \& Thompson (1991) in their study of Cygnus OB2, is somewhat larger than the standard 0.72 . However, it is in better agreeement with the distribution of points in the $(B-V),(U-B)$ diagram, since a shallower reddening vector with a slope of 0.72 would leave many more stars in a region inaccessible by a reddened star with normal intrinsic colours. We note in any case that the precise choice of the value of $E[U-B] / E[B-V]$ has virtually no impact on our results concerning the detection of early-type stars. The total-to-selective extinction ratio that we have adopted, $R_{\mathrm{V}}=A_{\mathrm{V}} / E[B-V]=3.1$, is the classical one and very close to the one used by Massey \& Thompson (1991), $R_{\mathrm{V}}=3.0$, for Cygnus OB2. The latter is in turn a roundoff of the value obtained by Torres-Dodgen et al. (1991), $R_{\mathrm{V}}=3.04 \pm 0.09$, which is virtually indistinguishable from the classical value given the uncertainty.

The bulk of stars in the field have positions in the colourcolour diagram suggesting intrinsic colours in the range of the kink of the solid curve (corresponding to spectral types between early $\mathrm{A}$ and early $\mathrm{G}$ ), reddened by $A_{\mathrm{V}} \sim 2 \mathrm{mag}$. Their magnitudes are consistent with their location at typical distances of $\sim 2 \mathrm{kpc}$, in the near end of the SagittariusCarina arm. A tail of redder stars, most probably cool luminous giants at varying distances and reddenings, is also clearly visible. However, the most remarkable feature related to our project is the loose clump of stars roughly centered on $(B-V) \simeq 1.7,(U-B) \simeq 0.3$, a position that is accessible only to reddened stars of the earliest types. Their location can also be characterized by the classical reddening-free parameter 


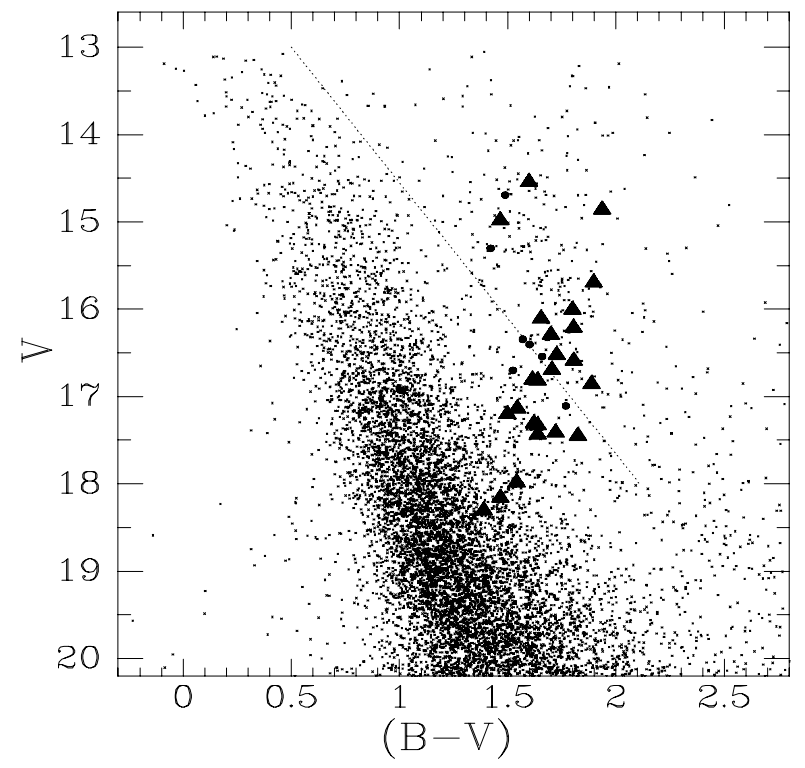

Fig. 2. Color-magnitude diagram showing the positions of all the nonsaturated stars in our field. The dashed line is a reddening vector whose length corresponds to the displacement of a star obscured by $A_{\mathrm{V}}=5$ mag. Stars with intrinsically blue colours having $Q<-0.7$ are noted as filled circles, and the proposed WRA 751 cluster members listed in Table 1 are plotted as filled triangles.

$Q=(U-B)-0.80(B-V)\left(\right.$ Johnson \& Morgan 1953) ${ }^{2}$, where the stars in this clump are characterized by $Q<-0.7$, roughly corresponding to a main-sequence spectral type $\mathrm{B} 3$ or earlier.

Figure 2 shows the position of the stars in the $(B-V), V$ diagram, with the stars having $Q<-0.7$ marked with special symbols. The $\sigma(B-V), \sigma(U-B)<0.2$ condition prevents us from identifying fainter stars that may fulfill the $Q$-criterion, and the cutoff at faint $V$ magnitudes is thus most likely not real.

\section{The WRA 751 cluster}

\subsection{Imaging}

In our initial EMMI observations we identified 20 stars fulfilling the $Q<-0.7$ criterion. Their spatial distribution clearly revealed their clustering around WRA 751, as 13 of them were found within a radius of only $76^{\prime \prime}$ from WRA 751, i.e., less than $3 \%$ of the field imaged with EMMI.

The deeper FORS2 observations expand this initial census up to the 24 blue stars in the surroundings of WRA 751 that we list in Table 1. Their positions are plotted in Fig. 3. These stars, marked as triangles in Fig. 2, are distributed around $B-V \simeq 1.67$, which corresponds to an average extinction $A_{\mathrm{V}} \simeq 6.1$ towards the cluster. Their relatively narrow range of colours indicates that the extinction within the cluster is rather small, probably not exceeding $A_{\mathrm{V}_{\text {int }}} \sim 1.5 \mathrm{mag}$. Most of the blue objects listed in Table 1 and plotted in Fig. 3 are projected

\footnotetext{
${ }^{2}$ Our definition follows the usual convention in current literature, which reverses the sign of the original definition by Johnson \& Morgan (1953); see e.g. Massey \& Thompson (1991). The coefficient multiplying $(B-V)$ is $E[U-B] / E[B-V]$ for which, as explained above, we prefer to adopt a value of 0.80 rather than the classical 0.72 .
}

within a distance of $117^{\prime \prime}$ of WRA 751, and the radius of the cluster is approximately $1^{\prime}$. Four additional stars are seen at larger distances, up to almost $4^{\prime}$ from WRA 751. The fairly strong degree of concentration towards WRA 751 of the other members suggests that these stars may not be associated to the cluster. However, both their colours and their magnitudes are within the range defined by the early stars closer to WRA 751, and we therefore consider them as possibly related to it, also given the rarity of stars with such photometric characteristics in the rest of the field covered by our observations. We have thus included them in our census listed in Table 1.

The observed $V$ vs. $Q$ diagram provides an approximation to the H-R diagram of the cluster, and is presented in Fig. 4. Evolutionary tracks for stars of different initial mass as given by LeJeune $\&$ Schaerer (2001) in Case $e$ (with high mass loss and no rotation, see Meynet et al. 1994) are also plotted. Tracks corresponding to initial masses of 9, 12, 20 and $25 M_{\odot}$ are traced with a dashed line, while those for $M_{i}=40$ and $60 M_{\odot}$ are shown with a dotted and a dot-dashed line, respectively. The additional long-dashed line represents the evolutionary track for $M_{i}=120 M_{\odot}$. All the tracks have been scaled to a distance of $6 \mathrm{kpc}$ (see Sect. 5) and reddened by $A_{\mathrm{V}}=6.1$ (see Sect. 4.1). Their corresponding Zero-Age Main-Sequence (ZAMS) is traced with a solid line. The large errors in the horizontal direction, mainly due to the faintness of the stars in $U$, prevent us from identifying accurate evolutionary phases and initial masses, and only spectroscopy can remove this degeneracy. Nevertheless, Fig. 4 indicates that the cluster stars can be quite massive, spaning a range between 9 and $120 M_{\odot}$ (the upper limit of the evolutionary tracks), where the lower limit of $9 M_{\odot}$ is due to the adopted constraint of $Q<-0.7$.

\subsection{Spectroscopy}

The spectra of the five OB candidates observed with FORS1 in July 2003 are shown in Fig. 5, where the stellar continuum has been normalized to unity. The stars ID number is taken from Table 1.

A number of absorption lines typical of the $\mathrm{O}$ and B spectral types can be easily identified: $\mathrm{H} \gamma$, HeI $\lambda 4471$, HeII $\lambda \lambda 4200,4686$, CIII $\lambda \lambda 4070,4650$ and OII $\lambda 4367$. The feature in absorption at $\lambda 4428$ is due to a diffuse interstellar band (DIB); its strength does not significantly vary among the observed stars, thus indicating quite similar reddenings.

The spectra were classified independently by each author via comparison with the spectral atlases of Walborn \& Fitzpatrick (1990) and Hanson (2003). The mean spectral types derived for the targets are listed in Table 2, ranging from asearly-as $\mathrm{O} 8$ to as-late-as B1. We estimate our classification to be accurate at less than 1.5 subtypes and two luminosity classes. We used the intrinsic $U B V$ colour terms given by FitzGerald (1970) as a function of spectral type and luminosity class, and derived from the observed photometry of the targets their extinction (in Table 2). This turns out to be in quite good agreement with the extinction estimated from the photometry alone (see Sect. 4.1). 
Table 1. Likely members of the WRA 751 cluster.

\begin{tabular}{rcccccc}
\hline \hline ID & $\alpha(2000)$ & $\delta(2000)$ & $V$ & $(U-B)$ & $(B-V)$ & $Q$ \\
\hline 1 & $11: 08: 37.6$ & $-60: 42: 46$ & $14.54 \pm 0.01$ & $0.31 \pm 0.04$ & $1.59 \pm 0.00$ & $-0.96 \pm 0.04$ \\
2 & $11: 08: 37.8$ & $-60: 42: 49$ & $16.81 \pm 0.01$ & $0.51 \pm 0.15$ & $1.63 \pm 0.02$ & $-0.79 \pm 0.15$ \\
3 & $11: 08: 38.0$ & $-60: 42: 35$ & $17.30 \pm 0.01$ & $0.21 \pm 0.16$ & $1.62 \pm 0.03$ & $-1.08 \pm 0.16$ \\
4 & $11: 08: 39.9$ & $-60: 42: 15$ & $18.30 \pm 0.02$ & $0.13 \pm 0.23$ & $1.38 \pm 0.05$ & $-0.97 \pm 0.24$ \\
5 & $11: 08: 40.1$ & $-60: 42: 18$ & $14.98 \pm 0.01$ & $0.13 \pm 0.05$ & $1.46 \pm 0.01$ & $-1.03 \pm 0.05$ \\
6 & $11: 08: 41.2$ & $-60: 42: 34$ & $16.29 \pm 0.01$ & $0.52 \pm 0.12$ & $1.69 \pm 0.01$ & $-0.83 \pm 0.12$ \\
7 & $11: 08: 41.6$ & $-60: 43: 18$ & $16.85 \pm 0.01$ & $0.80 \pm 0.19$ & $1.88 \pm 0.02$ & $-0.70 \pm 0.19$ \\
8 & $11: 08: 41.6$ & $-60: 42: 41$ & $14.85 \pm 0.01$ & $0.59 \pm 0.07$ & $1.93 \pm 0.01$ & $-0.95 \pm 0.07$ \\
9 & $11: 08: 41.7$ & $-60: 43: 01$ & $18.15 \pm 0.02$ & $0.10 \pm 0.21$ & $1.46 \pm 0.04$ & $-1.07 \pm 0.22$ \\
10 & $11: 08: 41.8$ & $-60: 42: 43$ & $17.41 \pm 0.01$ & $0.64 \pm 0.22$ & $1.72 \pm 0.03$ & $-0.72 \pm 0.22$ \\
11 & $11: 08: 42.4$ & $-60: 42: 57$ & $16.52 \pm 0.01$ & $0.54 \pm 0.13$ & $1.72 \pm 0.02$ & $-0.83 \pm 0.14$ \\
12 & $11: 08: 42.8$ & $-60: 42: 53$ & $17.45 \pm 0.01$ & $0.60 \pm 0.23$ & $1.82 \pm 0.03$ & $-0.85 \pm 0.23$ \\
13 & $11: 08: 43.1$ & $-60: 42: 50$ & $16.00 \pm 0.01$ & $0.41 \pm 0.10$ & $1.79 \pm 0.02$ & $-1.02 \pm 0.11$ \\
14 & $11: 08: 44.5$ & $-60: 42: 48$ & $16.59 \pm 0.01$ & $0.61 \pm 0.15$ & $1.80 \pm 0.02$ & $-0.83 \pm 0.15$ \\
15 & $11: 08: 45.2$ & $-60: 42: 57$ & $16.80 \pm 0.01$ & $0.42 \pm 0.14$ & $1.61 \pm 0.02$ & $-0.86 \pm 0.14$ \\
16 & $11: 08: 45.2$ & $-60: 42: 52$ & $17.43 \pm 0.01$ & $0.40 \pm 0.19$ & $1.63 \pm 0.03$ & $-0.90 \pm 0.19$ \\
17 & $11: 08: 46.0$ & $-60: 43: 00$ & $17.20 \pm 0.01$ & $0.36 \pm 0.15$ & $1.49 \pm 0.02$ & $-0.83 \pm 0.16$ \\
18 & $11: 08: 46.0$ & $-60: 42: 07$ & $17.32 \pm 0.01$ & $0.54 \pm 0.19$ & $1.63 \pm 0.03$ & $-0.76 \pm 0.19$ \\
19 & $11: 08: 46.4$ & $-60: 41: 58$ & $17.13 \pm 0.01$ & $0.35 \pm 0.15$ & $1.54 \pm 0.02$ & $-0.88 \pm 0.15$ \\
20 & $11: 08: 46.8$ & $-60: 42: 16$ & $16.10 \pm 0.01$ & $0.36 \pm 0.10$ & $1.65 \pm 0.02$ & $-0.95 \pm 0.10$ \\
21 & $11: 08: 48.9$ & $-60: 44: 23$ & $15.69 \pm 0.01$ & $0.59 \pm 0.19$ & $1.89 \pm 0.05$ & $-0.92 \pm 0.28$ \\
22 & $11: 08: 49.4$ & $-60: 43: 25$ & $16.21 \pm 0.01$ & $0.47 \pm 0.22$ & $1.80 \pm 0.06$ & $-0.96 \pm 0.24$ \\
23 & $11: 08: 50.9$ & $-60: 42: 46$ & $17.98 \pm 0.01$ & $0.47 \pm 0.24$ & $1.54 \pm 0.04$ & $-0.75 \pm 0.24$ \\
24 & $11: 09: 05.7$ & $-60: 42: 21$ & $16.69 \pm 0.01$ & $0.44 \pm 0.14$ & $1.70 \pm 0.02$ & $-0.91 \pm 0.14$ \\
\hline & & & & & & \\
\hline
\end{tabular}

The dereddened observed $(B-V)$ colours were then used to determine the effective temperature ( $T_{\text {eff }}$, in Table 2$)$ of the targets via the empirical calibrations of Slesnick et al. (2002). Although these calibrations are not very sensitive to $T_{\text {eff }}$ of $\mathrm{O}$ and early B stars, and do not take into account the effect of stellar wind blanketing, they extend to the B1I spectral type which is not included in the most up-to-date temperature scales published by Repolust et al. (2004) and Massey et al. (2005). Nevertheless, we have reported in Table 2 the $T_{\text {eff }}$ values given by Massey et al. (2005) for stars earlier than BOI. The difference between these two sets of effective temperatures is less than or comparable to the $1 \sigma$ errors on our spectral classification (see Sect. 5). For this reason and for the sake of homogeneity, we adopt the effective temperatures given by the empirical calibrations of Slesnick et al. throughout Sect. 5 and in Fig. 6.

\section{Discussion}

Our observations reveal the presence of a cluster of blue stars spatially coincident with WRA 751. Given the extreme paucity of early-type stars (with $Q<-0.7$ ) in the observed field, we identify this cluster as the birth-site of WRA 751. A chance alignment of a cluster of massive stars with a LBV is extremely unlikely, given that both classes of objects are rare.

Various distance estimates can be found in literature for WRA 751. van Genderen et al. (1992) derived a distance larger than 4-5 kpc from the photometry of field stars around WRA 751. This is consistent with the more recent determination of $6 \pm 1 \mathrm{kpc}$ (Nota, private communication) based on the radial velocity of WRA 751 and its surrounding HII region. We have adopted the latter value in this paper; we prefer it over a distance simply derived from the spectral types in Table 2 and the available photometry in Table 1 , since it is well known that the absolute magnitude of early-type stars displays a large scatter within any given spectral type (Garmany 1990; Jaschek \& Gómez 1998). At this distance, the angular extent of the cluster translates into a radius of $3.4 \mathrm{pc}$, with WRA 751 close to the cluster centre. Assuming that all stars more massive than $9 M_{\odot}$ have been detected (cf. Fig. 4) and that the initial mass function of the cluster is represented by a Miller \& Scalo's (1979) law, we roughly estimate the cluster mass to be $2.2 \times 10^{3} M_{\odot}$, i.e. about one order of magnitude larger than the Trapezium cluster in Orion (Herbig \& Terndrup 1986).

The H-R diagram of the observed five OB stars in the WRA 751 cluster is plotted in Fig. 6 . The $1 \sigma$ errors on $T_{\text {eff }}$ simply reflect the uncertainty of our spectral classification, while those on $M_{\mathrm{V}}$ take also into account the uncertainty on the adopted distance, the photometric errors and the uncertainty in $R_{\mathrm{V}}$ (see Sect. 3). The evolutionary tracks (dashed lines) are taken from LeJeune \& Schaerer (2001; Case $e$ with high mass loss and no rotation, see also Meynet et al. 1994); their ZAMS is represented with a solid line. We have added to the observed OB stars WRA 751 itself, for which we have assumed a mean, observed magnitude $V=12.14$, an extinction $A_{\mathrm{V}}=5.58 \mathrm{mag}$ and an O9.5I spectral type (cf. Hu et al. 1990). The effective temperature of WRA 751 has been derived once again from the empirical calibrations of Slesnick et al.

The position of the targets in the $\mathrm{H}-\mathrm{R}$ diagram indicates that the observed $\mathrm{OB}$ stars span a mass range between 12 and $40 M_{\odot}$, although the uncertainty on our spectral 


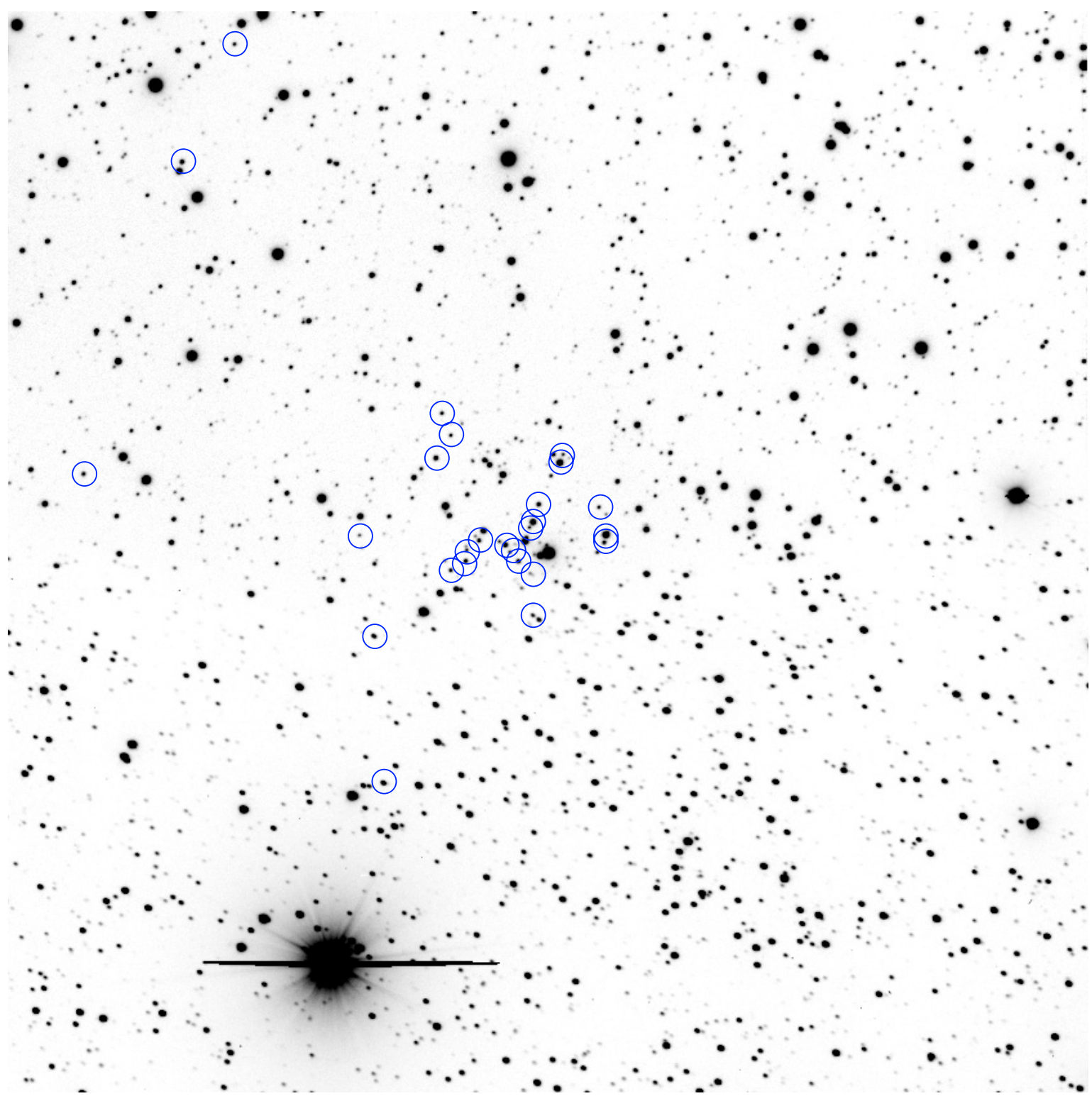

Fig. 3. A $B$-band image of the field around WRA 751, with the stars having $Q<-0.7$ marked (see Sect. 3). The field covers 7'06 $\times 7$ '06 and WRA 751 is at its center. North is up and East to the left.

Table 2. The spectral classification of five early-type stars in the WRA 751 cluster, their temperature and absolute magnitude. A distance of $6 \mathrm{kpc}$ is assumed.

\begin{tabular}{rccccc}
\hline \hline ID & $\begin{array}{c}\text { Spectral } \\
\text { type }\end{array}$ & $A_{\mathrm{V}}$ & $\begin{array}{c}\log \left(T_{\text {eff }}[\mathrm{K}]\right) \\
\text { (Slesnick et al. 2002) }\end{array}$ & $\begin{array}{c}\log \left(T_{\text {eff }}[\mathrm{K}]\right) \\
\text { (Massey et al. 2005) }\end{array}$ & $M_{\mathrm{V}}$ \\
\hline 1 & B0I & 5.67 & 4.37 & 4.47 & -5.02 \\
5 & O9V & 5.49 & 4.60 & 4.50 & -4.40 \\
8 & B1Ia & 6.57 & 4.25 & - & -5.61 \\
20 & O9I & 5.98 & 4.49 & 4.50 & -3.77 \\
22 & O8V & 6.54 & 4.60 & 4.53 & -4.22 \\
\hline
\end{tabular}

classification allows masses larger than $40 M_{\odot}$ in the case of the two O8V and O9V stars (\# 22 and 5 respectively). The progenitor of WRA 751 may have been as massive as $\sim 80 M_{\odot}$. Since the brighter and bluer stars were selected for the spectroscopic follow-up, we are confident that no star earlier than $\mathrm{O} 8$ is present in the cluster. If the absence of stars earlier than $\mathrm{O} 8$ were due to their evolution off the main sequence, this would set a lower limit on the age of the cluster of about $4 \mathrm{Myr}$, which turns out to be consistent with the current evolutionary phase of WRA 751. We note however that the absence of such early-type stars may also be simply due to an insufficient sampling of the upper end of the main sequence (i.e., small-number statistics) 


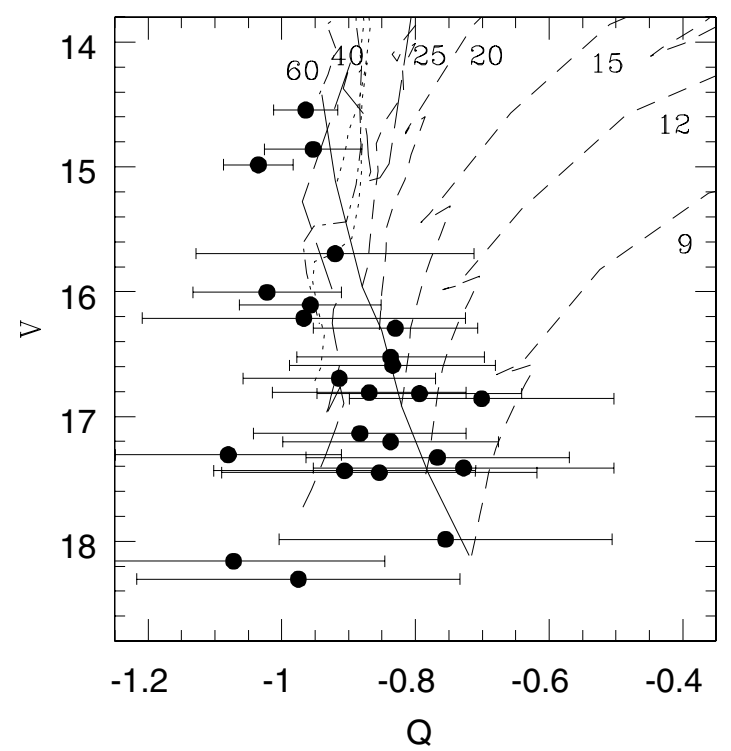

Fig. 4. The $V$ vs. $Q$ diagram of the intrinsically bluest stars in the observed fields (see text for definitions). The evolutionary tracks computed by LeJeune \& Schaerer (2001, Case $e$ with high mass loss and no rotation) for initial stellar masses of $9,12,15,20$ and $25 M_{\odot}$ are shown with dashed lines. The tracks for $M_{i}=40$ and $60 M_{\odot}$ are the dotted and dot-dashed lines, respectively. The additional long-dashed line represents the evolutionary track for $M_{i}=120 M_{\odot}$. All tracks have been scaled to a distance of $6 \mathrm{kpc}$ and reddened by $A_{\mathrm{V}}=6.1$ (see Sect. 4.1). The Zero-Age Main-Sequence (ZAMS) is traced with a solid line.

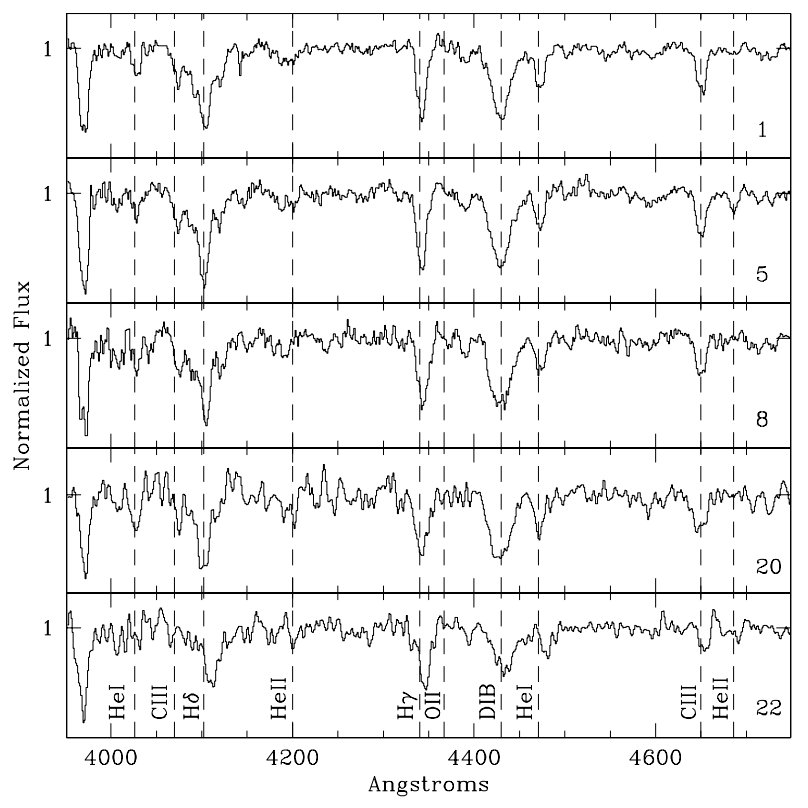

Fig. 5. The FORS1 spectra of five OB stars in the WRA 751 cluster. Their stellar continuum is normalized to unity. The most relevant lines for spectral classification are shown together with the diffuse interstellar band in absorption (DIB) at $\lambda \simeq 4430 \AA$.

given the limited mass that we estimate for the cluster. Thus, a younger age cannot be ruled out, although the classification of three late O/early B stars with luminosity class I argues for significant evolution implying an age of a few million years.

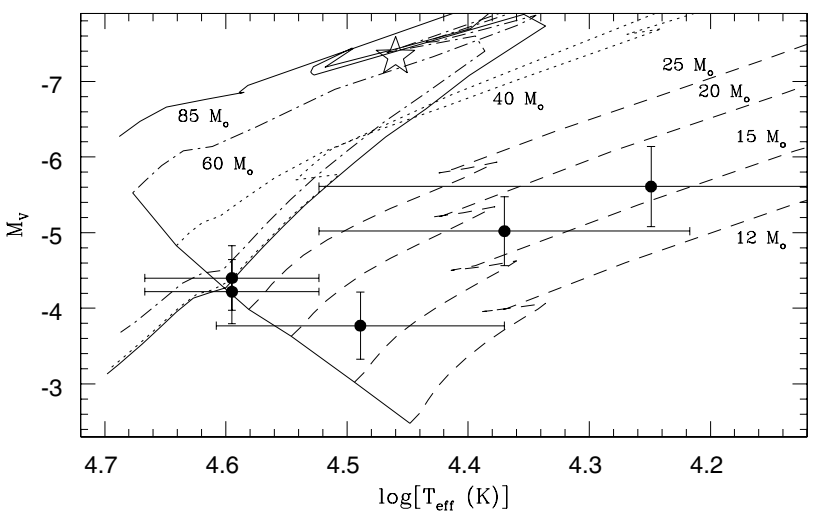

Fig. 6. The H-R diagram of the newly discovered $\mathrm{OB}$ stars around WRA 751, at an assumed distance of $6 \mathrm{kpc}$ (filled circles). The LBV star WRA 751 is shown with an open star symbol. Evolutionary tracks are from LeJeune \& Schaerer (2001, Case $e$ with high mass loss and no rotation). Tracks corresponding to initial masses of 12, 15, 20 and $25 M_{\odot}$ are traced as dashed lines, while those referring to $M_{i}=40$, 60 and $85 M_{\odot}$ are shown with a dotted, dot-dashed and solid line, respectively.

The data presented here offer a first glimpse of the nature of the birth-cluster of WRA 751, and need to be complemented with additional observations, leading to an accurate determination of its distance, stellar content and age. Its identification and study are an important step towards our understanding of the evolution of WRA 751 into the Luminous Blue Variable phase.

Acknowledgements. We acknowledge an anonymous referee whose comments and suggestions improved the paper. We are pleased to thank the staff of the La Silla Observatory for their support during our NTT observations, especially Mr. Duncan Castex and Ms. Mónica Castillo. We also thank the ESO User Support Department for their valuable assistance in the preparation of our spectroscopic observations, the ESO Data Flow Operations Group for their delivery of a data package of excellent quality, and the Paranal Science Operations staff in charge of the execution of the Service Mode observations. The allocation of time for the follow-up spectroscopy by the ESO Director General Discretionary Time Committee is gratefully acknowledged.

\section{References}

Bessell, M. S. 1990, PASP, 102, 1181

Bruzual, A. G., \& Charlot, S. 2003, MNRAS, 344, 1000

Dekel, A., \& Silk, J. 1986, ApJ, 303, 39

Georgelin, Y. M., \& Georgelin, Y. P. 1976, A\&A, 49, 57

FitzGerald, M. P. 1970, A\&A, 4, 234

Garmany, C. D. 1990, in Properties of hot luminous stars, (San Francisco: ASP), 16

Hanson, M. M. 2003, ApJ, 597, 957

Herbig, G. H., \& Terndrup, D. M. 1986, ApJ, 307, 609

Hu, J. Y., de Winter, D., The, P. S., \& Perez, M. R. 1990, A\&A, 227, L17

Humphreys, R. M., \& Davidson, K. 1994, PASP, 106, 1025

Jaschek, C., \& Gómez, A. E. 1998, A\&A, 330, 619

Johnson, H. L., \& Morgan, W. W. 1953, ApJ, 117, 313

Landolt, A. U. 1992, AJ, 104, 340

Langer, N., Hamann, W.-R., Lennon, M. J., et al., 1994, A\&A, 290, 819 
Leitherer, C., Schaerer, D., Goldader, J. D., et al. 1999, ApJS, 123, 3

LeJeune, T., \& Schaerer, D. 2001, A\&A, 366, 538

Maeder, A., \& Meynet, G. 2000, ARA\&A, 38, 143

Massey, P., Waterhouse, E., \& DeGioia-Eastwood, K. 2000, AJ, 119, 2214

Massey, P., DeGioia-Eastwood, K., \& Waterhouse, E. 2001, AJ, 121, 1050

Massey, P., Puls, J., Bresolin, F., Kudritzki, R. P., \& Simon, T. 2005, ApJ, 627, 477

Massey, P., \& Thompson, A. B. 1991, AJ, 101, 1408

Meynet, G., Maeder, A., Schaller, G., Schaerer, D., \& Charbonnel, C. 1994, A\&AS, 103, 97

Miller, G. E., \& Scalo, J. M. 1979, ApJS, 41, 513
Nota, A. 1997, in Luminous Blue Variables: Massive Stars in Transition, ed. A. Nota, \& H. J. G. L. M. Lamers, ASP Conf. Ser., 120,83

Parker, Q. A. 1997, Pub. Astron. Soc. Austr., 14, 123

Repolust, T., Puls, J., \& Herrero, A. 2004, A\&A, 415, 349

Silva, D. R., \& Péron, M. 2004, in The ESO Messenger, 118, 2

Slesnick, C., Hillenbrand, L. A., \& Massey, P. 2002, ApJ, 576, 880

Stetson, P. B. 1987, PASP, 99, 191

Torres-Dodgen, A. V., Carroll, M., \& Tapia, M. 1991, MNRAS, 249, 1

Van Genderen, A. M., The, P. S., de Winter, D., et al. 1992, A\&A, 258, 316

Walborn, N. R., \& Fitzpatrick, E. L. 1990, PASP, 102, 379 